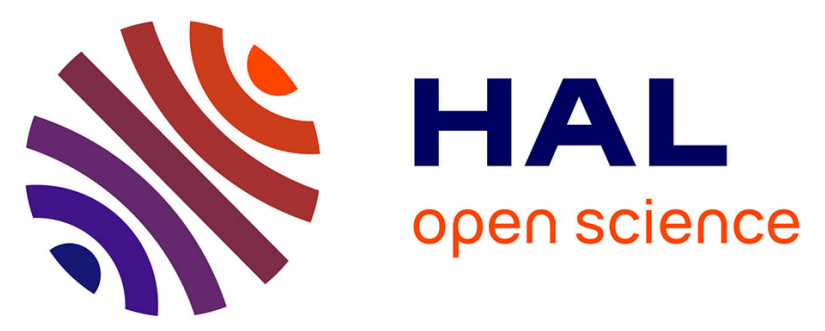

\title{
Absolute and regional cerebral perfusion assessment feasibility in head-down position with arterial spin-labeling magnetic resonance. A preliminary report on healthy subjects.
}

\author{
Aude Pavilla, Alessandro Arrigo, Sylvie Colombani, Mehdi Mejdoubi
}

\section{To cite this version:}

Aude Pavilla, Alessandro Arrigo, Sylvie Colombani, Mehdi Mejdoubi. Absolute and regional cerebral perfusion assessment feasibility in head-down position with arterial spin-labeling magnetic resonance. A preliminary report on healthy subjects.. Journal de Neuroradiologie / Journal of Neuroradiology, 2016. hal-01331444

HAL Id: hal-01331444

https://hal-univ-rennes1.archives-ouvertes.fr/hal-01331444

Submitted on 13 Jun 2016

HAL is a multi-disciplinary open access archive for the deposit and dissemination of scientific research documents, whether they are published or not. The documents may come from teaching and research institutions in France or abroad, or from public or private research centers.
L'archive ouverte pluridisciplinaire HAL, est destinée au dépôt et à la diffusion de documents scientifiques de niveau recherche, publiés ou non, émanant des établissements d'enseignement et de recherche français ou étrangers, des laboratoires publics ou privés. 


\section{ORIGINAL ARTICLE}

\section{Absolute and regional cerebral perfusion assessment feasibility in head-down position with arterial spin-labeling magnetic resonance on healthy subjects. Preliminary report.}

Aude Pavilla ${ }^{1,2}$, MSc, Alessandro Arrigo ${ }^{1}, \mathrm{MD}, \mathrm{PhD}$, Sylvie Colombani ${ }^{1}$, MD, Mehdi Mejdoubi ${ }^{1}, \mathrm{MD}, \mathrm{PhD}$

${ }^{1}$ Department of Neuroradiology, Pierre-Zobda-Quitman Hospital, University Hospital of Martinique, French West Indies, France; ${ }^{2}$ LTSI, INSERM UMR 1099, Université de Rennes 1,Rennes, F-35000, France.

Correspondence: Aude Pavilla, Department of Neuroradiology, Pierre-Zobda-Quitman Hospital, University Hospital of Martinique, French West Indies. Tel: +596 5965521 81; Fax: +596 5967516 68; Email: aude.pavilla@gmail.com 
ORIGINAL ARTICLE

\section{Absolute and regional cerebral perfusion assessment feasibility in head-down position with arterial spin-labeling magnetic resonance.A preliminary report on healthy subjects.}

Purpose: HD-positioning, used in some stroke centers during early ischemic stroke management, is empirical but supported by some physiological findings.It has been shown by non-MR methods that this position can increase cerebral perfusion. This study aimed to investigate magnetic resonance imaging (MRI) ability to measure the response to head down tilt (HDT) challenge in healthy volunteers. Cerebral blood flow (CBF) was assessed with arterial spin labeling (ASL)in supine and $\mathrm{HD}\left(-15^{\circ}\right)$ positions.

Methods: Cerebral perfusion was measured in supine and HD positions in seven healthy subjects at $1.5 \mathrm{~T}$ with a large magnet bore $(70 \mathrm{~cm})$ MR device. 3D-pseudo-continuous arterial spin-labeling (pCASL) sequences were acquired in both positions and cerebral blood-flow (CBF) maps were reconstructed. Regions of interest were: subcortical grey matter structures (Accumbens nuclei, Amygdala, Caudate nucleus, Hippocampus, Pallidum, Putamen and Thalamus), whole cortical grey matter andwhole white matter.

Results: White matter and subcortical gray matter structures CBF, averaged over the volunteers' sample, remained stable from supine to HD position. Accumbens nuclei and cortical grey matter CBF decreased by $11.5 \%(\mathrm{P}=0.013)$ and $11.4 \%(\mathrm{P}=0.018)$ when head position was changed from flat to $-15^{\circ}$.

Conclusions: RegionalCBF assessment especially in HDT is challenging with most perfusion techniques because of ionizing radiations, inherent limitations and logistical considerations. This preliminary report presents a non-invasive technique assessing regional and absolute cerebral blood flow changesin response to posture change. It can lead to further clinical investigations for a better understanding of cerebral perfusion.

Keywords: ASL; cerebral perfusion; head down; MRI. 


\section{INTRODUCTION}

CBF quantification is challenging. Different techniques have been used to assess cerebral perfusion directly or indirectly withthe gold standard being ${ }^{15} \mathrm{O}-\mathrm{PET}^{1,2}$ (positron emission tomography). However, because of radiation exposure and its high cost, the availability of this modality is limited.

The most common noninvasive technique for $\mathrm{CBF}$ indirect assessment is transcranialDoppler ${ }^{3}$, ${ }^{4}$ (TCD), which provides a good temporal resolution. With TCD, blood-flow velocity can be continuously monitored in the main supply arteries, usually the middle cerebral artery (MCA). The vessel cross-sectional area is the proportionality coefficient between flow and velocity. However, TCD can only assess relative and hemispheric CBF changes. Moreover velocity is measured only in the MCA trunk while microvascular blood flow in deep cerebral parenchyma is poorly assessed. Near infrared spectroscopy (NIRS) ${ }^{5}$ and more recently diffuse correlation spectroscopy ${ }^{6}$ are also noninvasive and allow continuous monitoring of deoxyhemoglobin and oxyhemoglobinin cortical regions. Like TCD, NIRS anddiffuse correlation spectroscopycan assess cerebral perfusion during position changes but, again, it only estimates relative changes.Imaging techniques like single photon-emission computed tomography(SPECT), positron-emission tomography $(\mathrm{PET})^{7}$ and perfusion computed tomography $(\mathrm{CT})^{8}$ can evaluate $\mathrm{CBF}$, but continuous $\mathrm{CBF}$ measurement or repeated assessments within brief intervals $(<24$ hours $)$ are not possible, because of contrast-agent persistence or potential side effects.

Magnetic resonance (MR)-perfusion techniques visualize global or regional absolute CBF and correlate positively with TCD and ${ }^{15} \mathrm{O}-\mathrm{PET}^{1,9}$.

However, due to small-bores, MRI was so far restricted to supine position anddid not allow position changes.Moreover, the first MR-perfusion assessment technique was done through dynamic susceptibility contrast (DSC) ${ }^{11}$, requiring an intravenous contrast agent based on gadolinium chelates.Gadolinium persistence in vessels prevents repeated CBF assessments at brief intervals. Arterialspin-labeling (ASL) is an MR technique providing images of absolute regional CBF without contrast-agent injection, used in perfusion $\operatorname{assessment}^{12-13}$.

HD-positioning, used in some stroke centers during early ischemic stroke management, is empirical but supported by some physiological findings. In acute ischemic stroke patients, this position may be used to increase cerebral perfusion ${ }^{6,10}$. Most studies dealing with posture influence on cerebral perfusion usually 
investigate global effect on perfusion and assess relative cortical perfusion ${ }^{6,10}$. The aim of this preliminary study was to assess the feasibility ofregional and global CBF changes measurements with ASL MRI in head down (HD) position.

\section{MATERIALS AND METHODS}

\section{Subjects}

Seven healthy subjects (2 women, 5 men) were enrolled. Mean age was 33.4 \pm 7.7 (range 26-42) years. They all underwent a physical examination and gave their informed consent. All were nonsmokers, normotensive, without diabetes and none was on medication. No caffeine or alcohol intake was allowed the day of the MRI and, for women of procreation age, the test was performed soon after their menstrual periods. The protocol was approved by the local ethics committee and received institutional review board agreement. Our procedures were in accordance with the Helsinki Declaration of 1975 (and as revised in 1983).

\section{Head Down Tilt Protocol}

Angles of $0^{\circ}$ and $-15^{\circ}$ were sequentially evaluated. Measurements at $0^{\circ}$ (supine) were considered as the baseline against which HD position $\left(-15^{\circ}\right)$ angle was compared. Supine position was carried out before HD position. To achieve the HD position, the subject was carefully positioned on a customized foam mattress (with $40^{\circ}$ angulation over $20 \mathrm{~cm}$ at one end; Figure 1), avoiding neck extension or compression of jugular veins or carotid arteries, with a mean $-15^{\circ}$ angle between the upper thorax and head. The head was approximately $20 \mathrm{~cm}$ below the heart, whereas the lower body remained flat. In each position, a 3D-ASL sequence was obtained after 4 minutes of equilibration.

\section{Cerebral Blood Flow Measurement}

MRI measurements were obtained with a large bore $(70 \mathrm{~cm})$ 1.5-T MR magnet (MR450w, GE, Milwaukee, WI, USA). We used a standard head-neck-spine array (8-channel head coil).

Axial T1 weighted images were acquired using a 3D gradient echo (GRE) sequence for segmentation purpose. 3DT1 images were acquired in supine position. Acquisition parameters were set as following: 
repetition time (TR) / echo time (TE):10.796 ms/ $4.4 \mathrm{~ms}$, inversion time (TI): $500 \mathrm{~ms}$, Bandwidth: $\pm 88,79$ $\mathrm{kHz}$, field of view (FOV) : $240 \times 240 \mathrm{~mm}^{2}$, matrix size was $256 \mathrm{x} 288$, in plane resolution: $0.94 \times 0.83 \mathrm{~mm}^{2}$ and slice thickness $1.4 \mathrm{~mm}$ for a total acquisition time of 2 minutes.

3D-FSE (fast spin echo) pseudo-continuous ASL (pCASL)sequences were obtained in both positions with following parameters: flip angle, $155^{\circ}$; TR/TE, $4554 \mathrm{~ms} / 10.7 \mathrm{~ms}$; labeling time, $1525 \mathrm{~ms}$; post-labeling delay, $1525 \mathrm{~ms}$; Distance between labeling and image plane: $10 \mathrm{~cm}, \mathrm{FOV}, 240 x 240 \mathrm{~mm}^{2}$; matrix, 512 points x 8 arms (spiral acquisition); slice thickness, $4 \mathrm{~mm}$; no gap; 60 images (30 control and 30 labeled), $4 \mathrm{~mm}^{3}$ isotropic resolution, $\mathrm{NEX}=3$, for a total acquisition time of $4 \mathrm{~min}$ and $28 \mathrm{~s}$. The image acquisition plane was the same in both positions.

\section{Post-processing}

For pCASL processing, subtracted raw images were post-processed using GE Readyview software on an Advantage Windows 4.6 workstation (GE Medical Systems), generating CBF maps in units of milliliters per 100 grams per minute.CBF quantification was calculated as follows using a conventional single compartment model as previously described ${ }^{12}$.

For each subject, T1 skull-stripped images were generated by running the FSL Brain Extraction Tool software (BET, http://www.fmrib.ox.ac.uk/fsl/bet2/index.html) on the high resolution T1 images. 3DT1 sequence was performed only in supine position and was then coregistered with ASL raw data in both supine and HD positions (after proper reorientation).

T1 images were segmented into three tissue classes (Figure 2) in its original resolution using FMRIB's Automated Segmentation Tool (FAST, http://www.fmrib.ox.ac.uk/fsl).

Grey matter (GM) and white matter (WM) binary masks (with a 0.9 threshold) were obtained for further quantitative analyzes on CBF maps thus limiting partial volume effects. Deep subcortical grey matter (scGM) structures segmentation was performed with FSL FIRST (FMRIB's Integrated Registration and Segmentation Tool). Accumbens nuclei, Amygdala, Caudate nucleus, Hippocampus, Pallidum, Putamen and Thalamus volumes of interest (VOIs) were obtained (Figure 2) and converted into binary masks for coregistation with CBF maps in supine position. A whole cortical GM (cGM) mask was also computed from those structures. The same processing protocol was applied for CBF maps in HD position. 


\section{Statistical Analysis}

Non normal data distribution was assumed and not tested owing to the low number of data. CBF changes were analyzed using nonparametric Wilcoxon rank tests with Statview software (SAS Institute v5.0, Cary, NC, USA). Following T1 images segmentation and coregistration with ASL, mean right-and-left subcortical GM (scGM), whole WM and whole cortical GM (cGM) VOIs were obtained.

Corresponding absoluteregional CBF valueswere compared between supine and HD positions. $P<0.05$ defined significance for all tests.

\section{RESULTS}

The HD position was clinically well-tolerated by all subjects.

In supine position, CBF was higher in cortical GM (cGM) than in subcortical GM (scGM) and WM (respectively $\mathrm{P}=0.028$ and $\mathrm{P}=0.018)$. $\mathrm{CBF}$ in scGM was significantly higher than in $\mathrm{WM}(\mathrm{P}=0.018)$.

From supine to HD position (Table 1$), \mathrm{CBF}$ declined significantly by $11.4 \pm 0.3 \%$ in cGM (P=0.018) and $11.5 \pm 0.3 \%$ in accumbens nuclei $(\mathrm{P}=0.013)$. $\mathrm{CBF}$ values remained stable in all other subcortical GM structures and WM. Among subcortical GM, pallidum had the lowest CBF values. Figure 3 illustrates significant cortical CBF variations in one subject.

\section{DISCUSSION}

ASL technique is a noninvasive tool to assess absolute and regional CBF. In supine position, $\mathrm{CBF}$ in WM was very low compared to other ROIs, but it is known that CBF varies among WM regions, from 33 to $45 \mathrm{~mL} / 100 \mathrm{~g} /$ minute according to Tarumi et $\mathrm{al}^{14}$. Higher CBF values were observed in cortical GM in comparison to subcortical GM regions as reported elsewhere in literature ${ }^{15}$. The lowest GM perfusion was found in pallidum as reported elsewhere due to its high concentration of iron shortening the local T1 ${ }^{15}$.

Recently, Favilla and al. ${ }^{6}$ measured with diffuse correlation spectroscopy an increase in cortical CBF associated with lower head of bed angle in acute ischemic stroke patients. It is generally assumed that this position may increase $\mathrm{CBF}^{6,10,16}$.

The current study demonstrates that measuring absolute regional CBF changes with head down tilt is 
feasible with ASL MRI. From supine to HD position, CBF remained steady in white matter and in most subcortical GM regions while simultaneously decreasing significantly in cortical GM $(-(11.4 \pm 0.3) \%)$ and accumbens nuclei $(-(11.5 \pm 0.3) \%)$ when averaged across healthy volunteers. Decreased perfusion in accumbens nuclei may be explained by anxiety accompanying the exam. It is welldocumented that those nuclei are involved in emotional and key-reward processing regions ${ }^{17}$.

This diminished CBF was also observed by Lucas etal $^{5}$, who measured a lowercortical oxygenationafter pharmacologically inducedchanges in mean blood pressure.

Unchanged perfusion in subcortical GM structures and white matterwith posture change can be explained by cerebral auto regulation processes as suggested in Gelinas et a1.study ${ }^{18}$. In this study, 21 healthy volunteers were randomly exposed to $90^{\circ} \mathrm{HD}$ and $90^{\circ}$ head-up positions. Theyobserved that MCA blood flow velocity remained stable, while mean arterial pressure increased significantly. They concludedthat perfusion was remarkably well-maintained.

Two quite recent MRI technological advances enabled the design of our study. First, ASL MR perfusionallows noninvasive repeated (but not continuous) cerebral perfusion measurements with regional, absolute and reproducible CBF quantification. Second, a large 70-cm magnet bore, available in commercial MR devices since 2005, allows moderate changes of the subject's position. HD positioning, despite minor discomfort, was well-tolerated by all subjects and we were able to acquire MR images.

Our pCASL sequence employed adiabatic labeling and background suppression pulses, a fast spin echo (FSE) acquisition, and a quantification approach based on a separate reference image to minimize, nonuniformities of the radiofrequency transmit and receive fields and the static magnetic field contribution.

Caution was made to place labeling plane orthogonally to large tagged arteries direction for similar labeling efficiency in both positions. Influence of head tilt on labeling efficiency could beinterestingly studied in future investigations.

Ourstudy is nevertheless only a preliminary report that should be completed on several axes. First, cerebral perfusion was evaluated only few minutes after HD-positioning, corresponding to an early cerebral autoregulation stage. Moreover, we studied here a rather small sample while CBF can be modified by other parameters such as age or sex.Supine position was systematically carried out before HD position which may represent a bias. This study may be completed by larger series considering individual variability and would 
also benefit from cerebrovascular reactivity co-investigation ${ }^{19}$ to establish correlations with CBF regional changes. Concerning regions of interest, we studied the overall cortical GM perfusion with HD positioning. Cortical segmentation, in order to distinguish each cortical territory specific response would be a further interesting topic.

\section{CONCLUSION}

We present a preliminary study that reports the feasibility of measuring CBF changes with HD positionbyMRI. White matter and subcortical gray matter structures perfusion remained unchanged with HD positioning whereas accumbens nuclei and cortical grey matter perfusion decreased significantly. The present study represents an initial step to explore regional and absolute cerebral blood flow changes in response to posture change with ASL. It could be of great interest for a better understanding of cerebral perfusion in physiologic and pathologic conditions.

\section{Disclosure of interest}

The authors declare that they have no conflicts of interest concerning this article. 


\section{REFERENCES}

1. Carroll TJ, Teneggi V, Jobin M, Squassante L, Treyer V, Hany TF et al. Absolute quantification of cerebral blood flow with magnetic resonance, reproducibility of the method, and comparison with $\mathrm{H}_{2}$ ${ }^{15}$ O positron emission tomography. J Cereb Blood Flow Metab 2002; 22: 1149-1156.

2. Grüner JM, Paamand R, Højgaard L, Law I. Brain perfusion CT compared with ${ }^{15} \mathrm{O}-\mathrm{H}_{2} \mathrm{O}-\mathrm{PET}$ in healthy subjects. EJNMMI Res 2011; 1: 28.

3. Aries MJ, Bakker DC, Stewart RE, De Keyser J, Elting JW, Thien T et al. Exaggerated postural blood pressure rise is related to a favorable outcome in patients with acute ischemic stroke. Stroke 2012; 43: 92-96.

4. Saeed NP, Horsfield MA, Panerai RB, Mistri AK, Robinson TG. Measurement of cerebral blood flow responses to the thigh cuff maneuver: a comparison of TCD with a novel MRI method. J Cereb Blood Flow Metab 2011; 31: 1302-1310.

5. Lucas SJ, Tzeng YC, Galvin SD, Thomas KN, Ogoh S, Ainslie PN. Influence of changes in blood pressure on cerebral perfusion and oxygenation. Hypertension 2010; 55: 698-705.

6. Favilla C, Mesquita R, Mullen M, Durduran T, Kim M, Minkoff D, Kasner S, Joel G, Yodh A and DetreJ ,Optical Bedside Monitoring of Cerebral Blood Flow in Acute Ischemic Stroke Patients During Head-of-Bed Manipulation,Stroke. 2014 ;45(5):1269-74.

7. Ito H, Kanno I, Takahashi K, Ibaraki M, Miura S. Regional distribution of human cerebral vascular mean transit time measured by positron emission tomography. Neuroimage 2003; 19: 1163-1169.

8. Mejdoubi M, Calviere L, Dumas H. Value of CT perfusion for the diagnosis of early middle cerebral artery stroke. J Radiol 2010; 91: 555-660.

9. Sorond FA, Hollenberg NK, Panych LP, Fisher NDL. Brainbloodflow and velocity: correlations between magnetic resonance imaging and transcranial Doppler sonography. J Ultrasound Med 2010; 29: $1017-1022$.

10. Schwarz, S., Georgiadis, D., Aschoff, A., \& Schwab, S. Effects of body position on intracranial pressure and cerebral perfusion in patients with large hemispheric stroke. Stroke, 33(2), 497-501, 2002.

11. Knutsson L, Ståhlberg F, Wirestam R., Absolute quantification of perfusion using dynamic susceptibility contrast MRI: pitfalls and possibilities, Magma. 2010 Feb;23(1):1-21.

12. Detre JA, Alsop DC.Perfusion magnetic resonance imaging with continuous arterial spin labeling: methods and clinical applications in the central nervous system. Eur J Radiol. 1999 May;30(2):11524.

13. Labriffe, M., TerMinassian, A., Pasco-Papon, A., N’Guyen, S., Aubé, C. (2015). Feasibility and validity of monitoring subarachnoid hemorrhage by a noninvasive MRI imaging perfusion technique: Pulsed Arterial Spin Labeling (PASL). Journal of Neuroradiology.

14. Tarumi T, Shah F, Tanaka H, Haley AP. Association between central elastic artery stiffness and cerebral perfusion in deep subcortical gray and white matter. Am J Hypertens 2011; 24: 1108-1113. 
15. Pfefferbaum A, Chanraud S, Pitel AL, Shankaranarayanan A, Alsop DC, Rohlfing T et al. Volumetric cerebral perfusion imaging in healthy adults: regional distribution, laterality, and repeatability of pulsed continuous arterial spin labeling (PCASL). Psychiatry Res 2010; 182: 266-273.

16. Nagatani K, Nawashiro H, Takeuchi S, Otani N, Wada K, Shima K. Effects of a head-down tilt on cerebral blood flow in mice during bilateral common carotid artery occlusion. Asian $\mathrm{J}$ Neurosurg 2012; 7: 171-173.

17. Wolf Dh, Pinkham AE, Satterthwaite TD, Ruparel K, Elliot MA, Valdez J, Smith MA, Detre JA, Gur RC, GurRE. Oral alprazolam acutely increases nucleus accumbens perfusion, Mol Psychiatry. 2013; 18(9): 960-961.

18. Gelinas JC, Marsden KR, Tzeng YC, Smirl JD, Smith KJ, Willie CK et al. Influence of posture on the regulation of cerebral perfusion. Aviat Space Environ Med 2012; 83: 751-757.

19. Boudiaf N, Attyé A, Warnking JM, Troprès I, Lamalle L, Pietras J, Krainik A. BOLD fMRI of cerebrovascular reactivity in the middle cerebral artery territory: A 100 volunteers' study. Journal of Neuroradiology, 2015. 
Table 1 Regional cerebral blood flows (in $\mathrm{mL} / 100 \mathrm{~g} / \mathrm{min}$ ) in supine and head-down positions

VOIs :

Subcortical Grey matter structures:

Accumbens nuclei

Amygdala

Caudate

Hippocampus

Pallidum

Putamen

Thalamus

Subcortical Grey matter

Cortical Grey matter

White matter
Position

$P^{b}$

\begin{tabular}{ccc} 
Supine $^{\mathrm{a}}$ & Head Down $^{\mathbf{a}}$ & \\
\hline $49.98 \pm 6.62$ & $44.25 \pm 6.75$ & 0.013 \\
$40.35 \pm 9.04$ & $39.94 \pm 4.64$ & 0.64 \\
$36.92 \pm 4.83$ & $35.52 \pm 4.45$ & 0.43 \\
$40.10 \pm 3.72$ & $39.79 \pm 5.04$ & 0.43 \\
$36.33 \pm 4.84$ & $35.46 \pm 4.29$ & 0.98 \\
$41.44 \pm 6.42$ & $40.16 \pm 5.31$ & 0.88 \\
$41.04 \pm 5.74$ & $39.62 \pm 6.61$ & 0.76 \\
$40.88 \pm 5.04$ & $39.25 \pm 4.66$ & 0.76 \\
$49.4 \pm 5,54$ & $43.76 \pm 7,98$ & 0.018 \\
$29.97 \pm 3.45$ & $29.69 \pm 4.96$ & 0.75
\end{tabular}

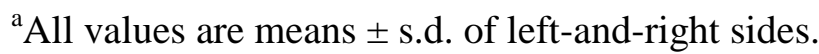

${ }^{b} P$ values were obtained by testing the mean of differences from 0 for paired series. 


\section{Titles and legends to figures}

Figure 1. Subject on the MRI table in a supine position (A) and after adding the customized foam mattress to raise the body and obtain $\mathrm{a}-15^{\circ}$ head-down position (B).

Figure 2. Binary masks derived for coregistration with CBF maps (A) WM ; (B) subcortical GM structures (clear blue: caudate; blue: pallidum; pink: putamen; green : thalamus); (C) cGM in native T1's space subject. Figure 3. Example of cerebral blood-flow variations between supine position (A) and head-down re-aligned to supine position (B) positions in a 26-year-old female subject.

The visual color scale indicates CBF (mL/min/100g) decrease in cortical GM $(-23 \%)$. 
Figure 1. Subject on the MRI table in a supine position (A) and after adding the customized foam mattress to raise the body and obtain $\mathrm{a}-15^{\circ}$ head-down position (B).

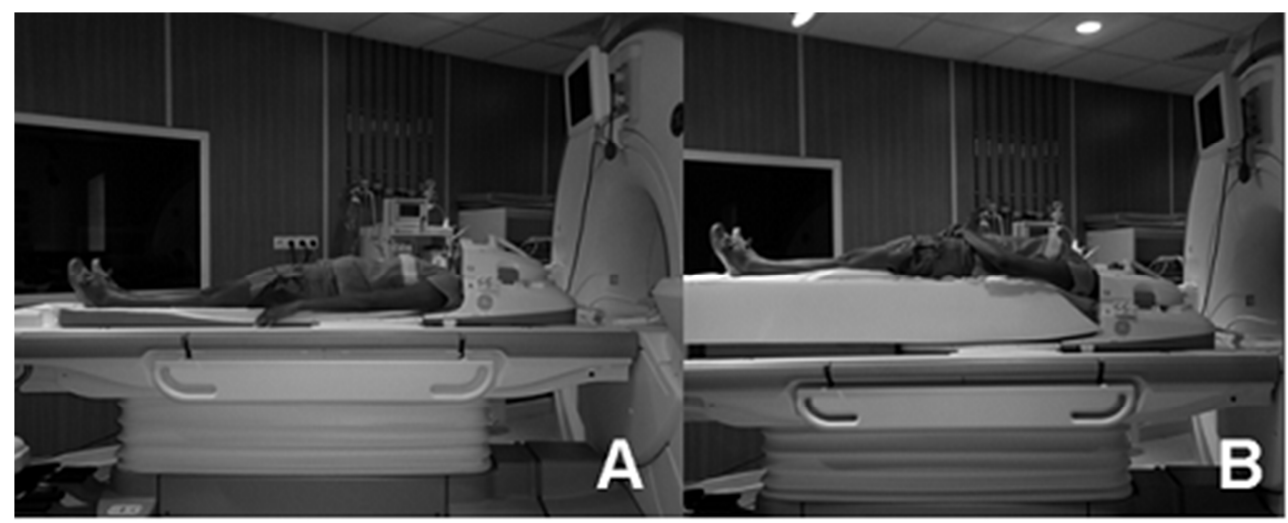

Figure l. Subject on the MRI table in a supine position (A) and after adding the customized

foam mattress to raise the body and obtain a $-15^{\circ}$ head-down position (B).

Figure 2. Binary masks derived for coregistration with CBF maps (A) WM ; (B) subcortical GM structures (clear blue: caudate; blue: pallidum; pink: putamen; green : thalamus); (C) cGM in native T1's space subject.
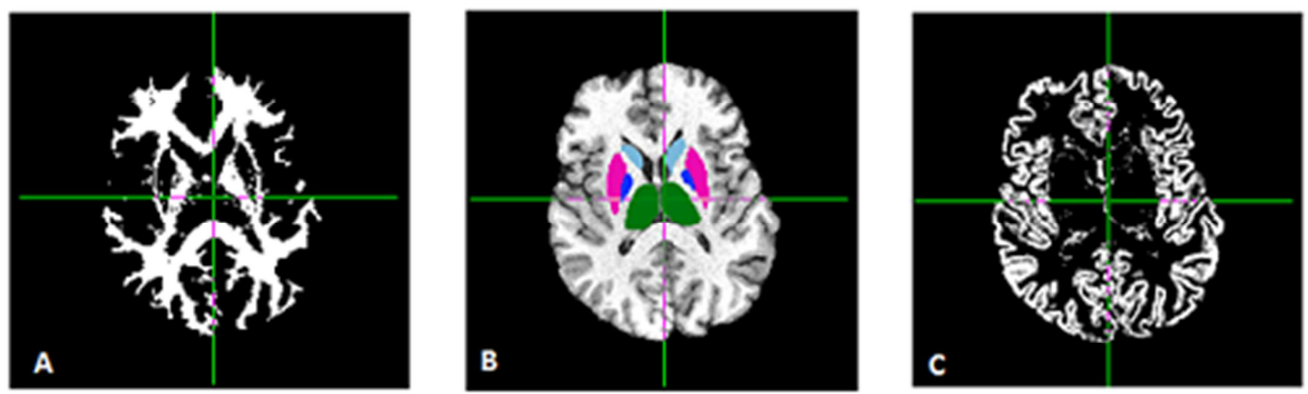

Figure 2. Binary masks derived for coregistration with CBF maps (A) WM; (B) subcortical

GMstructures (clear blve: cauda te; blve: pallidum; pink: putamen; green : thalamus); (C)

cGM in native T1's space ssbject. 
Figure 3. Example of cerebral blood-flow variations between supine position (A) and head-down re-aligned to supine position (B) positions in a 26-year-old female subject.

The visual color scale indicates $\mathrm{CBF}(\mathrm{mL} / \mathrm{min} / 100 \mathrm{~g})$ decrease in cortical GM (-23\%).

A
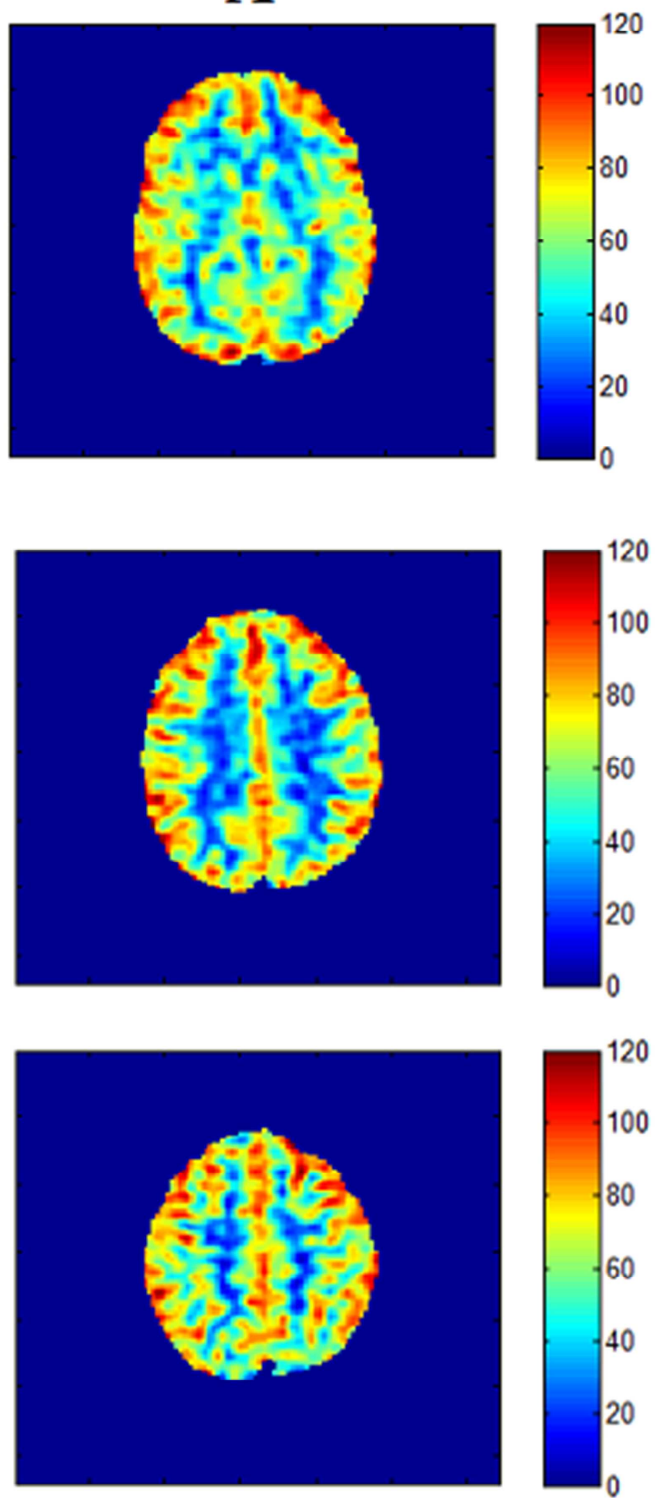

B
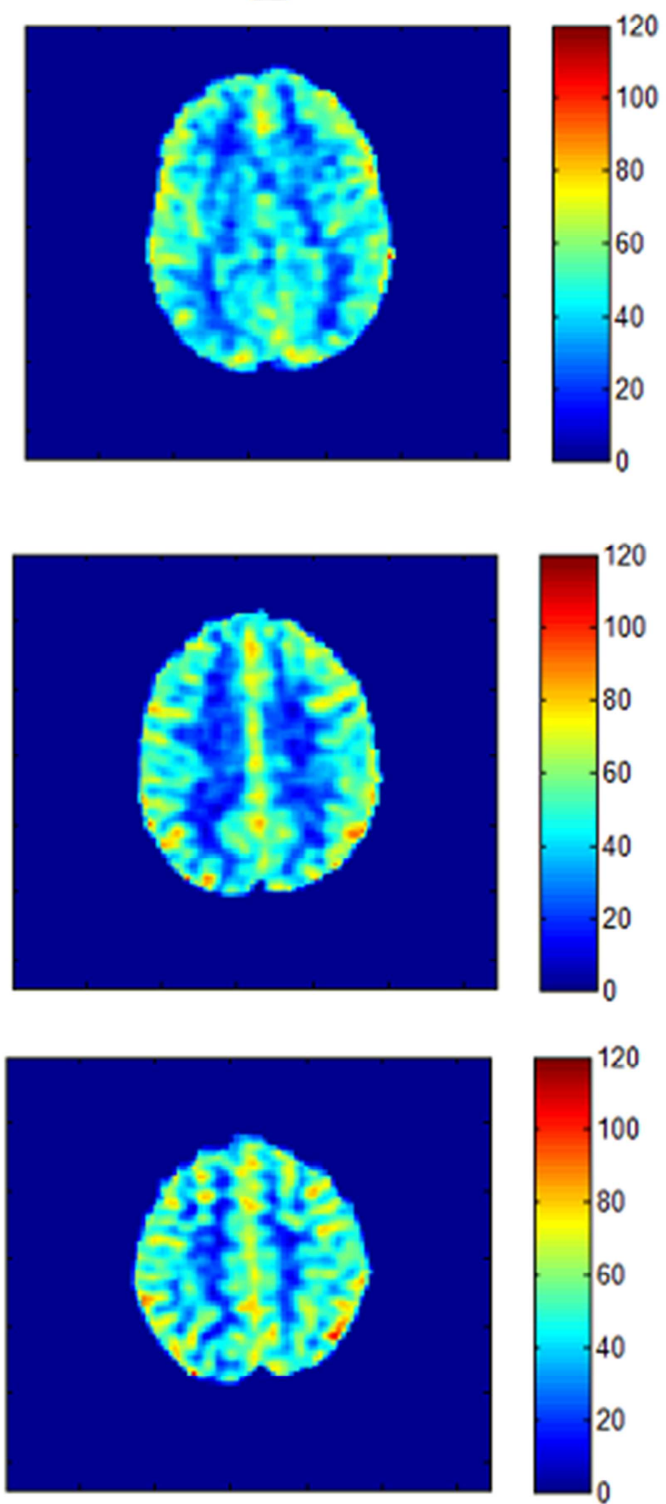

Figure 3. Example of cerebral blood flow variations betweeen supine $(A)$ and head-down realigned to supine position (B) positions in a 26 years-old female subject.

The visual colorbar indicates $\mathrm{CBF}(\mathrm{mL} / \mathrm{min} / 100 \mathrm{~g})$ decrease in cortical GM(-23\%). 
Table 2 Individual results for each VOI expressed in mean \pm standard deviation (sd) CBF (in mL/100g/min) in both supine and HD positions.

\begin{tabular}{|c|c|c|c|c|c|c|c|c|c|c|}
\hline & & Accumbens & Amygdala & Caudate & Hippocampus & Pallidum & Putamen & Thalamus & Cortical GM & White matter \\
\hline \multirow[t]{2}{*}{ Subject 1} & Supine & $52.82 \pm 0.62$ & $40,32 \pm 0.05$ & $38,11 \pm 0.47$ & $43,16 \pm 0,07$ & $35,02 \pm 0,78$ & $40,43 \pm 4,11$ & $45,69 \pm 2,41$ & $57,06 \pm 13,22$ & $30,84 \pm 10,45$ \\
\hline & Down & $52.11 \pm 2.86$ & $43,48 \pm 0.78$ & $39,40 \pm 0.25$ & $42,60 \pm 0,80$ & $39,06 \pm 2,91$ & $44,47 \pm 0,99$ & $45,78 \pm 0,57$ & $54,43 \pm 14,47$ & $32,78 \pm 11,14$ \\
\hline \multirow[t]{2}{*}{ Subject 2} & & & $32,77 \pm 4,47$ & $32,17 \pm 1.41$ & $34,82 \pm 1,84$ & $31,89 \pm 0,48$ & $35,10 \pm 1,19$ & $36,42 \pm 2,21$ & $41,97 \pm 12,61$ & $25,34 \pm 9,33$ \\
\hline & Head Down & $36.78 \pm 1.94$ & $34,8 \pm 4,21$ & $27,53 \pm 1.63$ & $33,52 \pm 2,61$ & $30,08 \pm 3,24$ & $32,86 \pm 2,80$ & $32,35 \pm 1,10$ & $35,99 \pm 11,30$ & $22,95 \pm 8,94$ \\
\hline \multirow[t]{2}{*}{ Subject 3} & Supine & $49.49 \pm 4.53$ & $38,98 \pm 1,41$ & $36,93 \pm 0,73$ & $42,16 \pm 0,66$ & $37,61 \pm 4,05$ & $41,97 \pm 2,77$ & $41,12 \pm 1,61$ & $53,79 \pm 14,17$ & $30,93 \pm 10,75$ \\
\hline & Head Down & & $39,43 \pm 0,85$ & $37,48 \pm 0,47$ & $40,00 \pm 1,81$ & $39,47 \pm 2,32$ & $43,92 \pm 0,26$ & $=0,73$ & $52,16 \pm 14,67$ & $29,75 \pm 10,85$ \\
\hline \multirow[t]{2}{*}{ Subject 4} & & & & & & & & & & 27,0 \\
\hline & Head Down & $47.80 \pm 2.36$ & $40,55 \pm 0,18$ & $38,95 \pm 3,01$ & $38,74 \pm 1,37$ & $38,90 \pm 0,47$ & $44,61 \pm 0,79$ & $38,45 \pm 1,83$ & $42,15 \pm 17,49$ & $36,91 \pm 16,02$ \\
\hline \multirow[t]{2}{*}{ Subject 5} & Supine & $61.79 \pm 9.51$ & $60,02 \pm 6,50$ & $46,85 \pm 2,09$ & $41,72 \pm 8,97$ & $46,06 \pm 3,91$ & $53,85 \pm 8,39$ & $46,97 \pm 1,85$ & $44.33 \pm 20,46$ & $36,06 \pm 12,42$ \\
\hline & Head Down & $46.16 \pm 8.64$ & $45,54 \pm 0,03$ & $36,31 \pm 0,88$ & $36,50 \pm 1,15$ & $33,43 \pm 3,26$ & $40,05 \pm 6,54$ & $36,73 \pm 2,26$ & $33,73 \pm 15,91$ & $27,33 \pm 9,43$ \\
\hline \multirow[t]{2}{*}{ Subject 6} & Supine & $49.44 \pm 2.07$ & $36,70 \pm 0,35$ & $37,02 \pm 1,85$ & $40,88 \pm 0,51$ & $33,13 \pm 1,77$ & $39,57 \pm 1,99$ & $44,67 \pm 0,17$ & $53,19 \pm 13,57$ & $30,86 \pm 12,42$ \\
\hline & Head Down & $43.92 \pm 2.50$ & $45,42 \pm 0,37$ & $38,04 \pm 0,62$ & $49,08 \pm 2,98$ & $36,76 \pm 1,77$ & $41,95 \pm 0,95$ & $50,09 \pm 1,10$ & $48,04 \pm 13,74$ & $33,23 \pm 11,94$ \\
\hline \multirow[t]{2}{*}{ Subject 7} & Supine & $47.22 \pm 0.01$ & $38,65 \pm 2,64$ & $34,73 \pm 0,07$ & $38,96 \pm 0,71$ & $35,30 \pm 1,15$ & $37,99 \pm 0,13$ & $35,71 \pm 1,22$ & $49,38 \pm 12,70$ & $28,76 \pm 10,44$ \\
\hline & Head Down & $35.52 \pm 0.01$ & $33,34 \pm 4,50$ & $30,95 \pm 0,28$ & $38,12 \pm 3,21$ & $30,52 \pm 0,66$ & $33,25 \pm 0,55$ & $31,79 \pm 0,14$ & $39.82 \pm 11,88$ & $24,92 \pm 9,64$ \\
\hline
\end{tabular}

